\title{
COUNTING THE FACES OF CUT-UP SPACES
}

\author{
BY THOMAS ZASLAVSKY
}

Communicated by Hans Weinberger, March 27, 1975

Let us take a topological space $X$ and "cut" it along certain subspaces $H_{1}$, $\ldots, H_{n}$. The connected components that remain we call the regions of the topological dissection of $X$ by the cut spaces $H_{1}, \ldots, H_{n}$.

How many regions are there? There is a large literature on this question for particular kinds of dissections. For instance much of the study of arrangements of hyperplanes in Euclidean and projective spaces has been concerned with counting regions, bounded regions, and lower-dimensional faces. As another example: into how many pieces is the plane cut by a given set of lines or curves? Answers have been found mainly for straight cuts in low dimensions or general position, some notable exceptions being Steiner's interest in circular and spherical cuts and Winder's and our work on arbitrary arrangements of hyperplanes. (For references see [1, Chapter 18], [2], [5], [6] .)

What has been lacking is a unified theory and the power to handle a great variety of dissections. In a forthcoming paper [6] we hope to provide such a theory. Here we shall mention most of the principal results and some areas of application.

1. The fundamental relations. The main idea of our theory is to count regions in terms of the set $L$ of intersections of the cuts or the set $L^{c}$ of connected components of intersections (we let $X$ be in both sets but $\varnothing$ be in neither). These sets are ordered by inclusion. Thus $L$ (and similarly $L^{c}$ ) has a Möbius function (cf. [3]), defined recursively by

$$
\mu(S, T)=\delta(S, T)-\sum\{\mu(S, U): S \subseteq U \subsetneq T\}, \quad(S, T) \in L^{2} .
$$

Here $\delta$ is the Kronecker delta. We also need the combinatorial Euler number of a space $X$, defined by $\kappa(X)=\chi-1$, where $\chi$ means the Euler characteristic of the one-point compactification of $X$. If $X$ is partitioned into $n_{k}$ open $k$-cells $(k=$ $0,1, \ldots, d)$, then $\kappa(X)=n_{0}-n_{1}+\cdots \pm n_{d}$.

A dissection $H$ of $X$ induces a dissection of $T \in L$ by $H_{T}=\{H \cap T: \varnothing \neq$ $H \cap T \neq T$ \}. The faces of $H$ are the regions of all the $H_{T}$ for $T \in L$.

THeOREM 1. Suppose $X$ is dissected by $H_{1}, \ldots, H_{n}$ into regions $R_{1}, \ldots$, $R_{m}(m \geqslant 0)$, and every face is a disjoint, finite union of open topological cells.

AMS (MOS) subject classifications (1970). Primary 05A15, 50B30; Secondary 06A35, $52 \mathrm{~A} 25,57 \mathrm{A65}, 57 \mathrm{C05}$.

Key words and phrases. Arrangement of hyperplanes, Dehn-Sommerville equations, partition of space, topological dissection, valuation of distributive lattice. 
Then

$$
\sum_{j=1}^{m} \kappa\left(R_{j}\right)=\sum_{U} \mu(U, X) \kappa(U)
$$

where $U$ is summed over $L$ or $L^{c}$, as desired, and $\mu$ is the corresponding Möbius function.

Equation (1) is the key to topological dissection theory. It is proved through Rota's theory of valuations of a distributive lattice [4]. A valuation on $D$ is a function which satisfies $\varphi(s)+\varphi(t)=\varphi(s \vee t)+\varphi(s \wedge t)$. Theorem 1 is a corollary of

THEOREM 2. If $D$ is a finite distributive lattice with a valuation $\varphi, P \subseteq D$ contains $0_{D}$ and all the join irreducibles of $D$, and $t \in P$ is not $0_{D}$ or join irreducible, then

$$
\sum\left\{\mu_{P}(s, t) \varphi(s): s \in P, s \leqslant t\right\}=0 .
$$

2. Properly cellular faces. If, for $T \in L^{c}$, all the regions of $T$ are open $(\operatorname{dim} T)$-cells, we say the dissection has properly cellular faces and denote by $f_{k}$ the number of $k$-dimensional faces.

THEOREM 3. If $\operatorname{dim} X=d$ and $X$ is dissected into properly cellular faces,

$$
\sum_{k=0}^{d} x^{d-k} f_{k}=(-1)^{d} \sum_{T \subseteq U} \sum_{\subseteq} \mu(U, T) \kappa(U)(-x)^{d-\operatorname{dim} T},
$$

where $T$ and $U$ are summed over either $L$ or $L^{c}$, as desired.

From (2) follow enumerations of the faces of an arrangement of hyperplanes in Euclidean or projective space, as in [5], or in any convex body; and topological generalizations. If the dissection has general position (any set of $k$ cuts meets in $\varnothing$ or a $(d-k)$-space $), \mu(U, T)=(-1)^{\operatorname{dim} T-\operatorname{dim} U}$, which greatly simplifies equation (2). Maximizing the number of faces often implies general position. Since also $\mu= \pm 1$ for a polytope dissected by its facets by [4], (2) yields the DehnSommerville equations, and generalizations such as to cubical polytopes.

3. In the sphere. If $X \subseteq S^{d}$ is dissected by subspaces $H_{1}, \ldots, H_{n}$ into regions $R_{1}, \ldots, R_{m}$, let $A=S^{d}-\left(R_{1} \cup \cdots \cup R_{m}\right)$ and let $\beta_{k}(A)$ be the $k$ th Betti number of $A$.

THEOREM 4. Assume that $d \geqslant 2 ; S^{d}-X$ and every face of the dissection of $X$ are disjoint, finite unions of open cells; and the regions are open in $S^{d}$. Then there are

$$
m=(-1)^{d}\left(\sum_{U} \mu(U, X) \kappa(U)-1+\beta_{0}(A)-\cdots \pm \beta_{d-2}(A)\right)
$$


regions, where $U$ is summed over $L$ or $L^{c}$, as desired.

The proof is by Theorem 1 and Alexander duality.

In open subsets of $S^{2}$ and $\mathbf{E}^{2}$, and in $\mathbf{P}^{2}$, we can generalize the classical enumerations for arrangements of lines and curves by using (3). For details see [6].

\section{REFERENCES}

1. Branko Grünbaum, Convex polytopes, Pure and Appl. Math., vol. 16, Interscience, New York, 1967. MR 37 \#2085.

2. - Arrangements and spreads, Conference Board of the Mathematical Sciences, Regional Conference Series in Math., no. 10, Amer. Math. Soc., Providence, R. I., 1972. MR 46 \#6148.

3. Gian-Carlo Rota, On the foundations of combinatorial theory. I. Theory of Möbius functions. Z. Wahrscheinlichkeitstheorie und Verw. Gebiete 2 (1964), 340-368. MR 30 \#4688.

4. - On the combinatorics of the Euler characteristic, Studies in Pure Math. (presented to Richard Rado), Academic Press, London, 1971, pp. 221-233. MR 44 \#126.

5. Thomas Zaslavsky, Facing up to arrangements: Face-count formulas for partitions of space by hyperplanes, Mem. Amer. Math. Soc. No. 154 (1975).

6. - A combinatorial analysis of topological dissections, Advances in Math. (to appear).

DEPARTMENT OF MATHEMATICS, MASSACHUSETTS INSTITUTE OF TECHNOLOGY, CAMBRIDGE, MASSACHUSETTS 02139 